\title{
Simulación numérica de la interacción fluido-estructura para predecir la respuesta de aerogeneradores sin palas a vibraciones inducidas por el viento en ciudades compactas
}

\author{
(Numerical simulation of fluid-structure interaction to predict \\ the response of bladeless wind turbines to wind-induced \\ vibrations in compact cities)
}

Cristian Andrade-Terán ${ }^{1}$

\begin{abstract}
Resumen
En este artículo se presenta un análisis de la respuesta fluido-estructura de los aerogeneradores sin palas que funcionan por resonancia aeroelástica inducida, los cuales pueden ser utilizados en ciudades o pueblos pequeños para formar parte de un urbanismo sostenible. En este análisis se llevan a cabo simulaciones numéricas del comportamiento del viento y del efecto denominado vórtices de von Karman que produce al rodear la estructura del aerogenerador utilizando como datos de entrada las velocidades del viento medidas por la Estación Meteorológica Mariscal Sucre en Quito. Las simulaciones CFD determinan la señal de excitación causada por los diferentes flujos de viento existentes. Asimismo, los efectos que estas oscilaciones causan en la estructura se simulan a través de un estudio modal y respuesta armónica a resonancia. Los resultados obtenidos muestran un aumento proporcional en la frecuencia y amplitud del desprendimiento de vórtices al aumento de la velocidad del viento, lo que provoca diferentes señales de excitación que hacen que el aerogenerador oscile con amplitudes entre 6 a $11 \mathrm{~cm}$. Finalmente, las simulaciones transitorias muestran que la presencia de casas y edificios en las cercanías en donde se instala el aerogenerador hacen que la dirección de la calle de vórtices varíe, así como las alteraciones en la frecuencia y amplitud de la excitación.
\end{abstract}

Palabras clave

Respuesta armónica, resonancia, sostenible, turbina eólica, vibración inducida.

\begin{abstract}
This article presents an analysis of the Fluid-Structure response of bladeless wind turbines that work by induced aeroelastic resonance, which can be used in cities or small towns to form part of sustainable urban planning. In it, numerical simulations of the behavior of the wind and the effect called Von Karman Vortices that it produces when surrounding the structure of the wind turbine are carried out, taking as input data the wind speeds measured by the Mariscal Sucre Meteorological Station in Quito. CFD simulations determine the excitation signal caused by the different existing wind flows, the effects that these oscillations cause on the structure are simulated through a modal study and harmonic response to resonance. The results obtained show a proportional increase in the frequency and amplitude of the vortex shedding to the increase in wind speed, causing different excitation signals that cause the wind turbine to oscillate with amplitudes between 6 and $11 \mathrm{~cm}$. Finally, the transient simulations show that the presence of houses and buildings in the vicinity where the wind turbine is installed causes the direction of the vortex street to vary, as well as alterations in the frequency and amplitude of the excitation.
\end{abstract}

\section{Keywords}

Wind turbine without blades, induced vibration, harmonic response, resonance, sustainable. 


\section{Introducción}

Una turbina eólica es un sistema que utiliza la turbulencia del viento para generar energía eléctrica. Convencionalmente, se han utilizado grandes estructuras con palas para absorber la energía eólica; sin embargo, hoy existen modelos de aerogeneradores sin palas, más pequeños y simples de instalar, que aprovechan el efecto de desprendimiento de vórtices generado por la dinámica del viento al evadir la estructura del aerogenerador. Por ejemplo, la empresa española Vortex Bladeless presentó una patente con varios modelos de turbinas eólicas que aprovechan exitosamente la energía eólica mediante la generación de vórtices inducidos a pequeña y mediana escala. (Yáñez, 2018).

En dinámica de fluidos, la generación de vórtices es un fenómeno bien estudiado. Esta ocurre cuando un flujo, ya sea laminar o turbulento, cruza un obstáculo. El desprendimiento de vórtices, también denominados vórtices de von Karman, induce o causa vibraciones en el eje transversal a la dirección del flujo. Estas fluctuaciones que dependen de la velocidad del fluido alcanzan una frecuencia estable que, al entrar en resonancia con la frecuencia natural de la estructura, hace que la estructura vibre durante mucho tiempo. En las construcciones civiles se intenta evitar a toda costa este efecto para prevenir fracturas o, al menos, que la estructura esté adaptada para soportar elásticamente las vibraciones. El viento es la carga estocástica que genera las calles de vórtices al cruzar estructuras. (Lopez, 2002; Saengsaen et al., 2019). En la actualidad se ha avanzado notablemente en modelos no lineales y en el estudio de las vibraciones en estructuras civiles. Dentro de este campo se encuentran los análisis modales en los que se emplean simulaciones numéricas para determinar la frecuencia natural que puede tener una determinada estructura, así como la respuesta a un estímulo externo que en superposición genera deformaciones o vibraciones, fenómeno conocido como resonancia (Karaağaçlı \& Özgüven, 2021; Peter \& Leine, 2017).

La energía que crea el viento por el desprendimiento de vórtices en la estructura es utilizada por el aerogenerador sin palas, mediante la transformación de la energía mecánica inducida por vibración, para obtener energía eléctrica a través de un alternador de imanes fijos. Las ventajas de este tipo de aerogenerador con respecto a los aerogeneradores con palas son principalmente su fácil instalación y mantenimiento, porque no está constituido por mecanismos complejos, sino que ocupa únicamente un mecanismo de imanes permanentes para la amortiguación del armónico inducido, lo que evita recurrir a sistemas de amortiguación más complicados y que requerirán un mayor mantenimiento (Yáñez, 2018, enero 23). Otra ventaja aparente es la distribución y composición de un conjunto de aerogeneradores, ya que en diferentes estudios se ha constatado que no influye de forma drástica colocarlos relativamente cerca, por lo tanto, se puede colocar varios de ellos para trabajar en conjunto (Saengsaen et al., 2019). A diferencia de los generadores convencionales, estos generan poca energía y están diseñados para ser utilizados cerca de donde se consumirá la energía, por lo que se instalan en áreas urbanas y en ciudades pequeñas.

Actualmente, los principios de sostenibilidad y los objetivos de desarrollo sostenible son fundamentales en cualquier tipo de proyecto, planificación y tecnología. Uno de estos objetivos está orientado a garantizar el acceso a una energía asequible, segura, sostenible y moderna para todos, y otro objetivo fundamental es adoptar medidas urgentes para combatir el cambio climático y sus efectos. Es decir, cualquier acción, por pequeña que sea, es de gran ayuda para 
lograr estos objetivos. Los aerogeneradores de vibración inducida son generadores de electricidad compactos y fáciles de instalar (diseñados para entornos urbanos) que junto con los paneles solares microhidroeléctricos pueden contribuir a la estrategia para formar ciudades sostenibles, con la cual se pretende urbanizar de manera compacta, mantener espacios verdes, reducir emisiones y utilizar fuentes de eficiencia energética y energía renovable para desarrollar ciudades compactas e inteligentes. (Andrade, 2021, julio 27; Comisión de Sustentabilidad Capbauno, 2020).

\section{Material y métodos}

Para entender y predecir el funcionamiento de un generador sin palas, que funciona aprovechando la energía que provoca el viento al producir vórtices en una estructura vertical, se ha estudiado el fenómeno tanto en la dinámica del fluido como en la respuesta de la estructura. Para realizar un análisis completo de la interacción fluido-estructura mediante simulación numérica, se ha llevado a cabo una modelación y simulación de las propiedades mecánicas y estructurales del aerogenerador, para determinar los modos naturales de vibración y el sistema de amortiguación necesarios para los diferentes flujos de viento. Asimismo, las simulaciones CFD ha servido para analizar los efectos del fluido (aire) sobre la estructura en condiciones ambientales medidas en una determinada zona de Sudamérica midiendo la frecuencia de desprendimiento de vórtice causado por el viento a su paso por un aerogenerador instalado. También se ha analizado la influencia del entorno donde se instalará para predecir su funcionamiento en ese entorno.

\subsection{Definición de la estructura}

Basada en el modelo propuesto por Vortex Bladeless (España Patente n. ${ }^{\circ}$ BR112013002403A, 2018) de $2.75 \mathrm{~m}$ de altura y $25-30 \mathrm{~cm}$ de diámetro, con velocidad de arranque de $3 \mathrm{~m} / \mathrm{s}$ y potencia nominal estimada de $100 \mathrm{~W}$ en condiciones óptimas, la estructura de la turbina eólica se modela digitalmente y su rigidez se ajusta para adaptarse a las condiciones de la región. En la figura 1 se ilustra el aerogenerador sin palas para el cual se harán los estudios. Se ha diseñado un modelo 3D para determinar aspectos de su composición y geometría a través de un análisis mecánico estructural y modal. La estructura está compuesta por una parte móvil de oscilación y dos fijas de amortiguación-sujeción. El mástil o parte móvil está diseñado a base de fibra de carbono de peso medio, y las zonas de sujeción de acero estructural, con un anclaje al suelo, el cual aumenta la rigidez de toda la estructura.

Mediante un análisis modal de elementos finitos con Ansys Mechanical APDL, se ha calculado, utilizando la geometría descrita en la figura 1 y las condiciones de borde al ser instalado, los modos de vibración natural de la estructura libremente sin aplicar ningún tipo de mecanismo o sistema externo de amortiguación. En la tabla 1 se presentan las frecuencias de vibración natural de la estructura en diez modos diferentes. 
Figura 1. Diagrama del aerogenerador de estudio basado en el modelo Tacoma
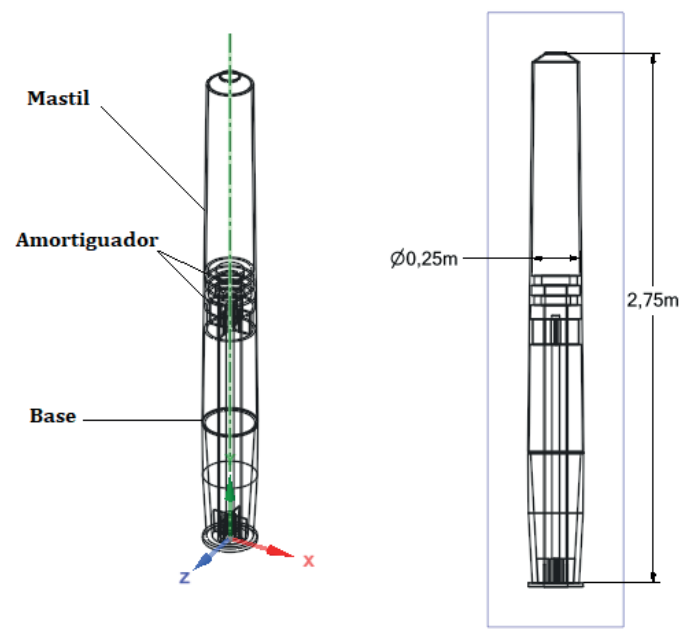

A partir de este estudio modal se ha podido estudiar la rigidez necesaria de la estructura, las condiciones mecánicas y los esfuerzos realizados en los diferentes modos. Posteriormente, se ha analizado la respuesta de la estructura a la resonancia provocada por la perturbación del viento al bordear el aerogenerador, introduciendo como entrada la frecuencia y la fuerza de sustentación que los vórtices desprenden en la estela posterior a la estructura. La ecuación fundamental utilizada para el estudio modal de la estructura libre de carga es Ec.1:

$[M]\{\ddot{u}\}+C\{\dot{u}\}+[K]\{u\}=\{0\}$

Donde:

$[M]$ : matriz de masas

$\{\ddot{u}\}$ : vector de aceleraciones

C:: amortiguamiento del sistema

$\{\dot{u}\}$ vector de velocidades

$[K]$ : matriz de rigidez

$u$ : desplazamientos

Tabla 1. Modos de vibración natural de la estructura

de la turbina eólica sin sistema de amortiguación

\begin{tabular}{|c|c|}
\hline Modo & Frecuencia $(\mathbf{H z})$ \\
\hline 1 & 1.177 \\
\hline 2 & 1.1865 \\
\hline 3 & 9.5002 \\
\hline 4 & 9.5764 \\
\hline 5 & 10.085 \\
\hline 6 & 99.831 \\
\hline 7 & 101. \\
\hline 8 & 135.96 \\
\hline 9 & 190.86 \\
\hline 10 & 235.16 \\
\hline
\end{tabular}


De la tabla 1, solo los modos 1 y 2 tienen vibraciones transversales con las que se puede trabajar para convertir el movimiento en energía eléctrica. Al variar la masa y la rigidez de la estructura, la frecuencia natural de oscilación se puede ajustar para aproximarse a la frecuencia de excitación generada por el desprendimiento de vórtices en la superficie cilíndrica de la turbina eólica. Los sistemas de amortiguación mecánica como resortes o imanes permanentes se han utilizado previamente para amortiguar o ajustar la frecuencia. En este trabajo se presentan las fuerzas necesarias por unidad de volumen en los soportes de amortiguación necesarios para ajustar la rigidez de la estructura con el fin de lograr resonancia con la excitación, y se valida con un estudio de respuesta asumiendo una excitación completamente armónica (Yáñez, 2018, junio 7; Y. Hu et al., 2018).

Los análisis de armónicos son utilizados para determinar la respuesta en estado estable de una estructura a cargas que varían sinusoidal o armónicamente con el tiempo, con lo que se puede predecir como esta estructura se comportará al entrar en resonancia (Ansys, 2020). Como se verá posteriormente la excitación provocada por el desprendimiento de vórtices ocurre de forma muy similar a un armónico por lo que se ha parametrizado esta señal como armónicos a diferentes frecuencias y amplitudes, dependiendo de la velocidad del flujo de viento, para simular sus efectos sobre el mástil del generador. Esta señal en superposición, cuando es igual a la frecuencia natural genera la resonancia que actuará sobre la estructura del aerogenerador haciéndola vibrar.

\subsection{Definición de fluidos}

Para caracterizar el flujo de viento que actúa en la región, se han utilizado los datos medidos durante 7 días por el centro de la estación meteorológica ubicada en el parque Bicentenario (antiguo Aeropuerto Mariscal Sucre), ubicado geográficamente en la latitud -0.225219, longitud: -78.5248 $0^{\circ} 13^{\prime} 31^{\prime \prime}$ sur, $78^{\circ} 31^{\prime} 29^{\prime \prime}$ oeste, en la zona ecuatorial del planeta. En la figura 2 se presenta la tabla proporcionada por Meteored (2021), para un periodo de 7 días en Quito, Ecuador, estación Mariscal Sucre. Las mediciones realizadas en los últimos días de agosto, en los cuales se registraron mayores velocidades de ráfagas en el viento, e históricamente es el periodo del año en el que existen mejores condiciones para aerogeneradores. Estas lecturas se hicieron a $10 \mathrm{~m}$ de la superficie terrestre, con una presión promedio de 540 mmHg y humedad del aire de entre 70 y $76 \%$.

Figura 2. Velocidad media, dirección y ráfagas de viento

$\begin{array}{llllllllllllllllllllllllllll}00 & 06 & 12 & 18 & 00 & 06 & 12 & 18 & 00 & 06 & 12 & 18 & 00 & 06 & 12 & 18 & 00 & 06 & 12 & 18 & 00 & 06 & 12 & 18 & 00 & 06 & 12 & 18\end{array}$

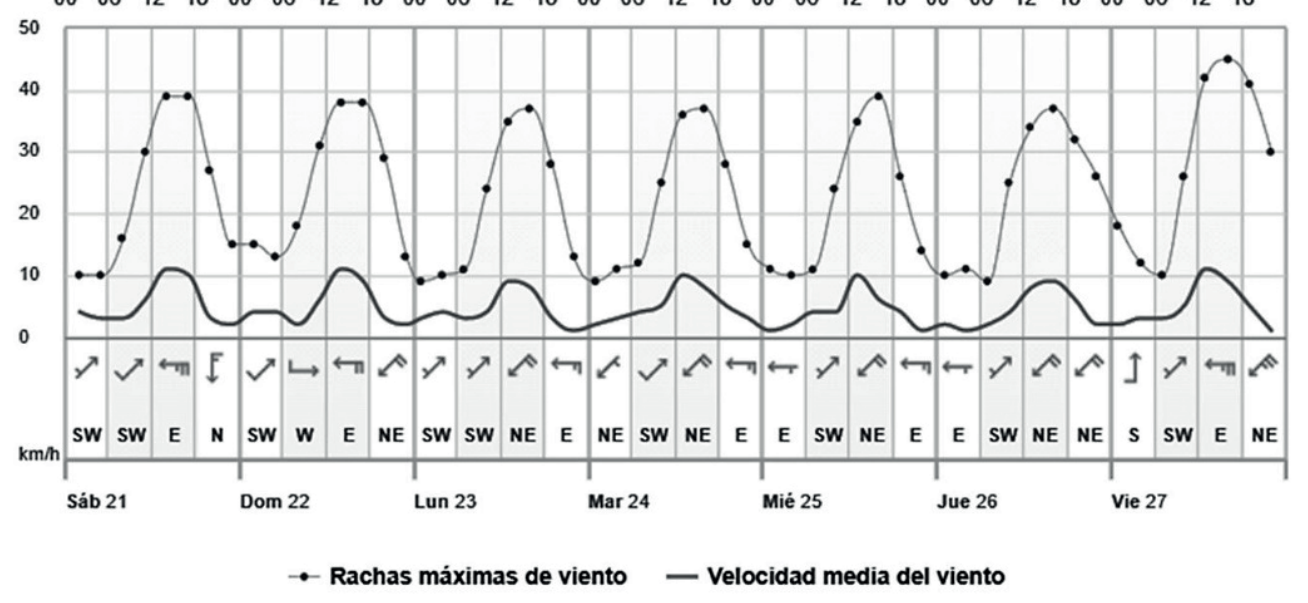


Los métodos de simulación de la dinámica de fluidos en régimen estacionario permiten establecer y predecir intensidades y dirección del viento actuante sobre el cañón o zona urbana, así como las turbulencias que causarán las obstrucciones de flujo causadas por edificaciones en las ciudades (Bustamante Campoverde, 2021). Utilizando Rwind, un software especializado para simular los efectos del viento sobre estructuras, que a su vez utiliza un solucionador OpenFoam, se han podido predecir los efectos de la interacción del fluido con un entorno urbano modelado con varias casas y pequeños edificios donde se pretende instalar aerogeneradores sin palas. El túnel de viento se ha definido con la magnitud de caudal medio más frecuente entre las 12 de la mañana y las 6 de la tarde en los 7 días consultados, aproximadamente $10 \mathrm{~km} / \mathrm{h}$ $(2,77 \mathrm{~m} / \mathrm{s})$, y se asume un perfil de viento para ciudades pequeñas con pocos edificios (Guevara Díaz, 2013). El perfil de viento está ilustrado en la figura 3.

Figura 3. Perfil eólico utilizado para el análisis en áreas urbanas

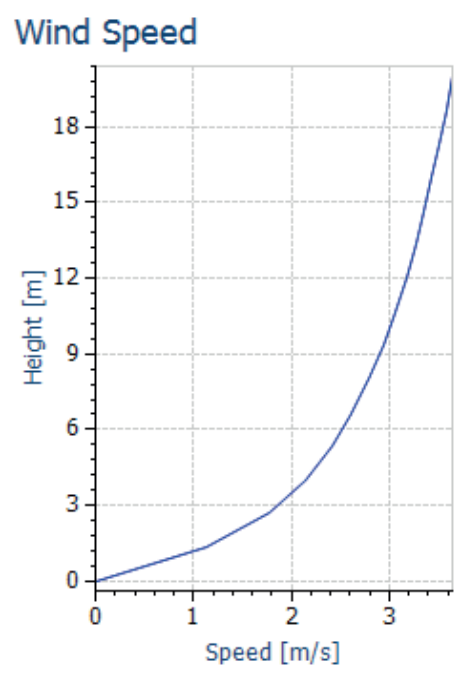

Tanto en la figura 4 como en la 5 se presentan contornos de velocidad del viento en la dirección delantera $\left(0^{\circ}\right)$ y la dirección trasera $\left(180^{\circ}\right)$. En estas pruebas se observaron notables variaciones en la magnitud y dirección del viento producidas por impactos en las superficies de edificios, lo cual genera turbulencias, a diferencia del flujo en campos abiertos. Por ello, se han analizado estos detalles con la simulación transitoria del fluido sobre la estructura.

Figura 4. Contornos de la velocidad en entorno urbano

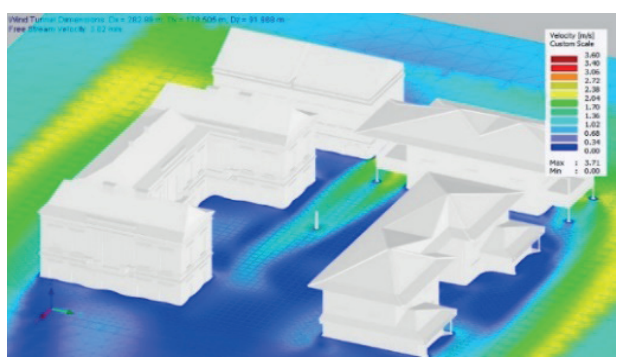

a)

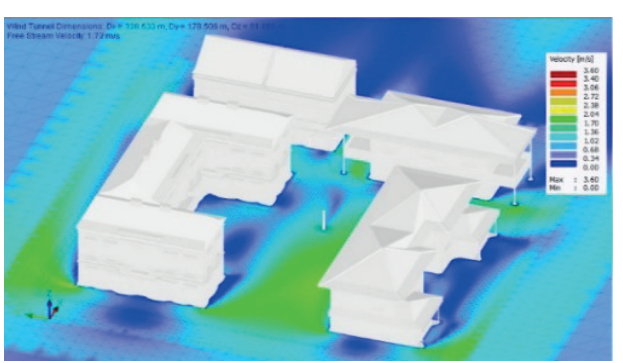

b)

Nota: a) dirección $0^{\circ}$, b) dirección $180^{\circ}$. 
Figura 5. Isolíneas de flujo de viento en entorno urbano

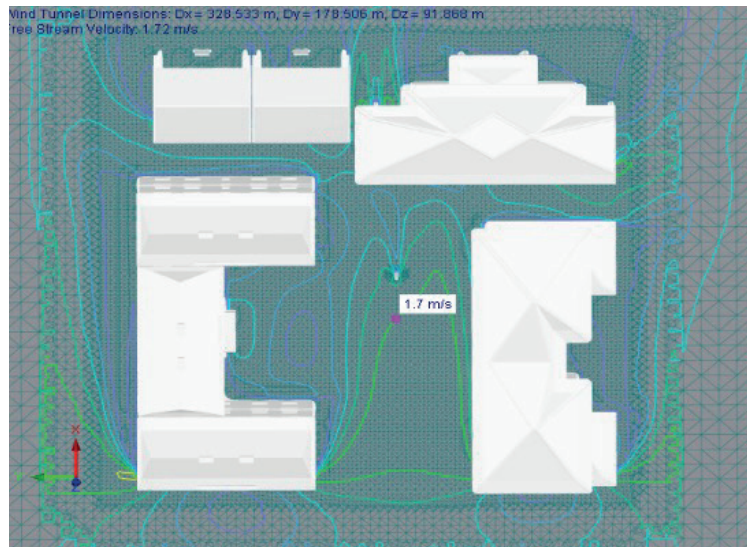

a)

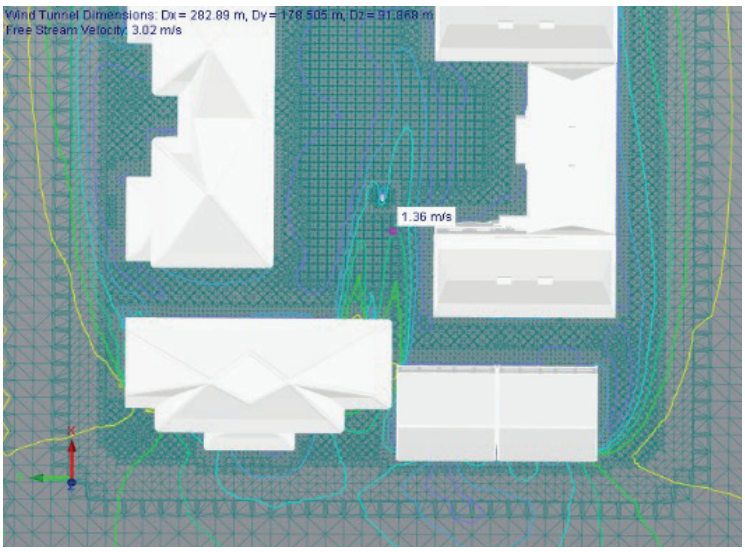

b)

Nota: a) dirección $0^{\circ}$, b) dirección $180^{\circ}$.

Para simular los efectos transitorios del viento en una sección del aerogenerador, se ha utilizado un entorno bidimensional con Ansys Fluent implementando un modelo de simulación de foucault desprendido (DES) que se ha adaptado al modelo Spalart-Allmaras (S-A) de una sola ecuación basada en la vorticidad, para predecir los efectos de la turbulencia a lo largo del tiempo. La función de transporte para el modelo se describe en la Ec.2. Se ha utilizado un método de solución SIMPLE y una formulación transitoria implícita de segundo orden estableciendo un tiempo entre pasos del orden $10^{-3}$ (Spalart \& Allmaras, 1992) en un lapso de 10 s para la velocidad media del viento y de $5 \mathrm{~s}$ para ráfagas de viento, donde se monitorean parámetros aerodinámicos como el coeficiente de arrastre y el coeficiente de elevación $(\mathrm{Cl})$ y se traza la fuerza de elevación, de modo que se pueda establecer la frecuencia con la que se liberan los vórtices desde la superficie de la turbina eólica. Se ha utilizado el modelo DES o simulación de remolino dependiente con modificación S-A, ya que en trabajos previos con simulación 2D de generación de vórtices se ha mostrado una mejor predicción de la frecuencia de desprendimiento (Saengsaen et al., 2019; Y. Hu et al., 2020).

$$
\frac{\partial}{\partial t}(\rho \tilde{v})+\frac{\partial}{\partial x_{i}}\left(\rho \tilde{v} u_{i}\right)=G_{v}+\frac{1}{\sigma_{\tilde{v}}}\left[\frac{\partial}{\partial x_{i}}\left\{(\mu+\rho \tilde{v}) \frac{\partial \tilde{v}}{\partial x_{j}}\right\}+C_{b 2} \rho\left(\frac{\partial \tilde{v}}{\partial x_{j}}\right)^{2}\right]-Y_{v}+S_{\tilde{v}}
$$

Donde:

$G_{v}$ : es la producción de viscosidad turbulenta

$Y_{v}$ : es la destrucción de la viscosidad turbulenta que se produce a la menta a la pared

$\sigma_{\mathfrak{v}}, C_{b 2}:$ son constantes $(0.66 ; 0.62)$

$v$ : viscosidad cinemática

$S_{\breve{v}}$ : término determinista

El flujo de viento se ha simulado tomando los valores presentados en la figura 2, para la velocidad media y las ráfagas alcanzadas en las horas punta. Además, se ha analizado las variaciones que se producen a causa de la interacción del viento en un entorno urbano obsta- 
culizado por edificaciones, como casas y edificios pequeños, así como un entorno de campo abierto sin obstáculos para fluir. Se asume que el aerogenerador está instalado de tal manera que interactúe con el flujo medido a 10 m por Meteored (2021) y se ha simulado para un valor medio del diámetro de la estructura de $0.275 \mathrm{~m}$. El mallado de campo 2D utilizado en las simulaciones transitorias CFD se muestra en la figura 6 para flujo libre y flujo obstaculizado respectivamente. Así se han definido dos ambientes diferentes con condiciones de contorno iguales para comparar el comportamiento del viento y la estela de vórtices que deja a su paso al rodear la estructura del aerogenerador.

Figura 6. Geometría de control 2D mallada para simulación transitoria de desprendimientos de vórtices

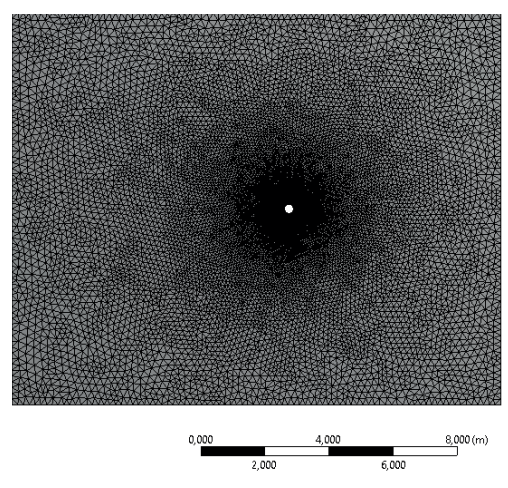

a)

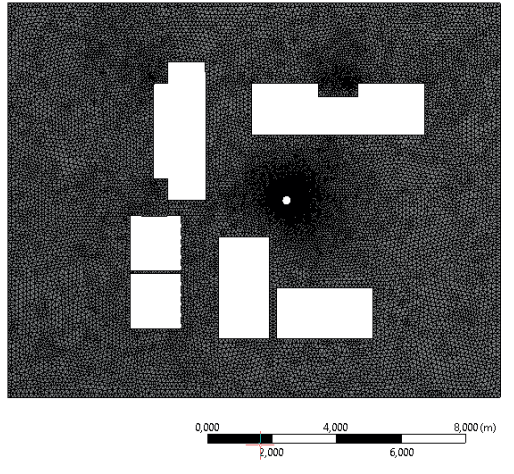

b)

Nota: a) campo abierto b) en entorno urbano

\section{Resultados y discusión}

\subsection{Respuesta estructural al estímulo del viento que fluye libremente}

Inicialmente se ha necesitado definir la señal de excitación provocada por el fluido, que en este caso es el viento, la cual causará la vibración en la estructura. Con base en las velocidades del viento descritas en los datos obtenidos en los 7 días consecutivos de agosto, las magnitudes de entrada han sido parametrizadas como condición de borde en la simulación CFD de túnel de viento transitorio. Primero, sin modelar ningún obstáculo al flujo del viento, se obtiene la curva de oscilación de fuerzas a través del tiempo sobre el eje transversal a la dirección de ataque del viento, y, mediante la transformada de Fourier, se convierte esta señal al dominio de la frecuencia para determinar las propiedades con la que el viento excitará a la estructura. A continuación, en la figura 7 se muestra la curva de oscilación de la fuerza de elevación que se crea y su respectiva transformada de Fourier para la velocidad media más frecuente, señal que se extiende similarmente a lo largo de todo el mástil. 
Figura 7. Fuerzas de soporte de la curva de oscilación y diagrama espectral para la velocidad media del viento

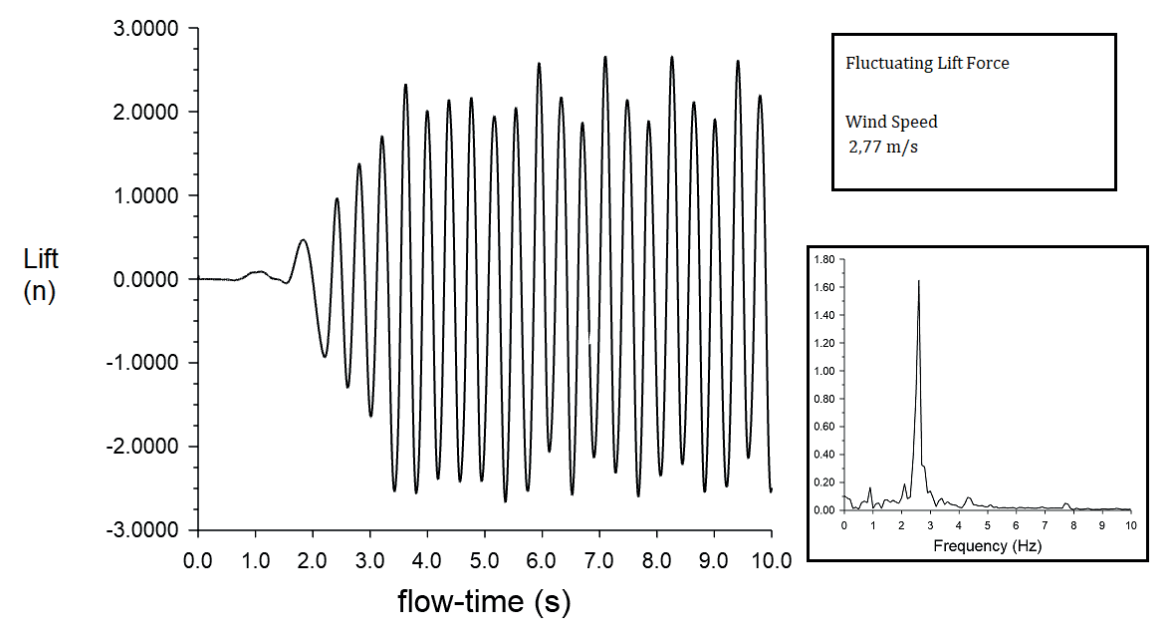

Con los datos obtenidos de la simulación, es posible determinar la frecuencia con la que los vórtices se desprenden en la estela dejada por el aire al rodear la estructura del aerogenerador y la dirección en que aparece la calle de vórtices. Para la velocidad media del viento con magnitud de $2.77 \mathrm{~m} / \mathrm{s}$, la frecuencia de desprendimiento es de $2.625 \mathrm{~Hz}$. Determinada la velocidad regular del viento en la zona donde se recogieron los datos en 7 días, se busca que esta sea la velocidad de entrada con la que funcione el aerogenerador sin palas. En la figura 8 se ilustra el desprendimiento de vórtices que se produce para un flujo de viento de $2.77 \mathrm{~m} / \mathrm{s}$ y Re= $5.2 \mathrm{E} 4$ alrededor de la superficie circular que representa al perímetro del aerogenerador; se puede ver la dirección constante en la que se desprenden los vórtices y como al alejarse de la estructura se desvanecen. I no existir ningún obstáculo alrededor, no hay cambios de dirección ni cambios bruscos en magnitud.

Figura 8. Contorno de vorticidad con caudal de viento de $2.77 \mathrm{~m} / \mathrm{s}$ en un ambiente libre

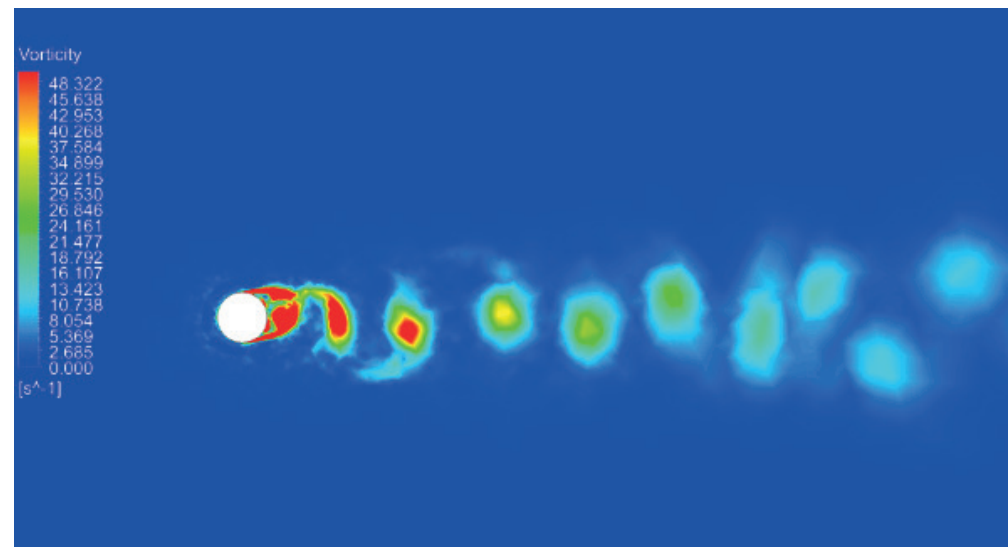

También se han analizado las fluctuaciones causadas por las ráfagas de viento más frecuentes en los datos consultados. En la figura 9 se presenta las curvas dependientes del tiempo y su respectivo análisis espectral para determinar la frecuencia de oscilación. Las velocidades del viento 
alcanzadas en las ráfagas también deben ser utilizadas y consideradas para el ajuste de la rigidez y puesta a punto de la estructura, ya que estas rachas están muy presentes a lo largo del día. Los diferentes caudales del viento causan una excitación fluctuante con su frecuencia de oscilación respectiva. Es destacable el aumento de la frecuencia y amplitud del desprendimiento de vórtices con el aumento de la velocidad de flujo del viento, lo cual genera una mayor fuerza de sustentación, fuerza que en resonancia hará que el aerogenerador vibre y, así, genere energía eléctrica.

Figura 9. Curvas de oscilación de las fuerzas de elevación y el espectro para ráfagas de viento más frecuentes

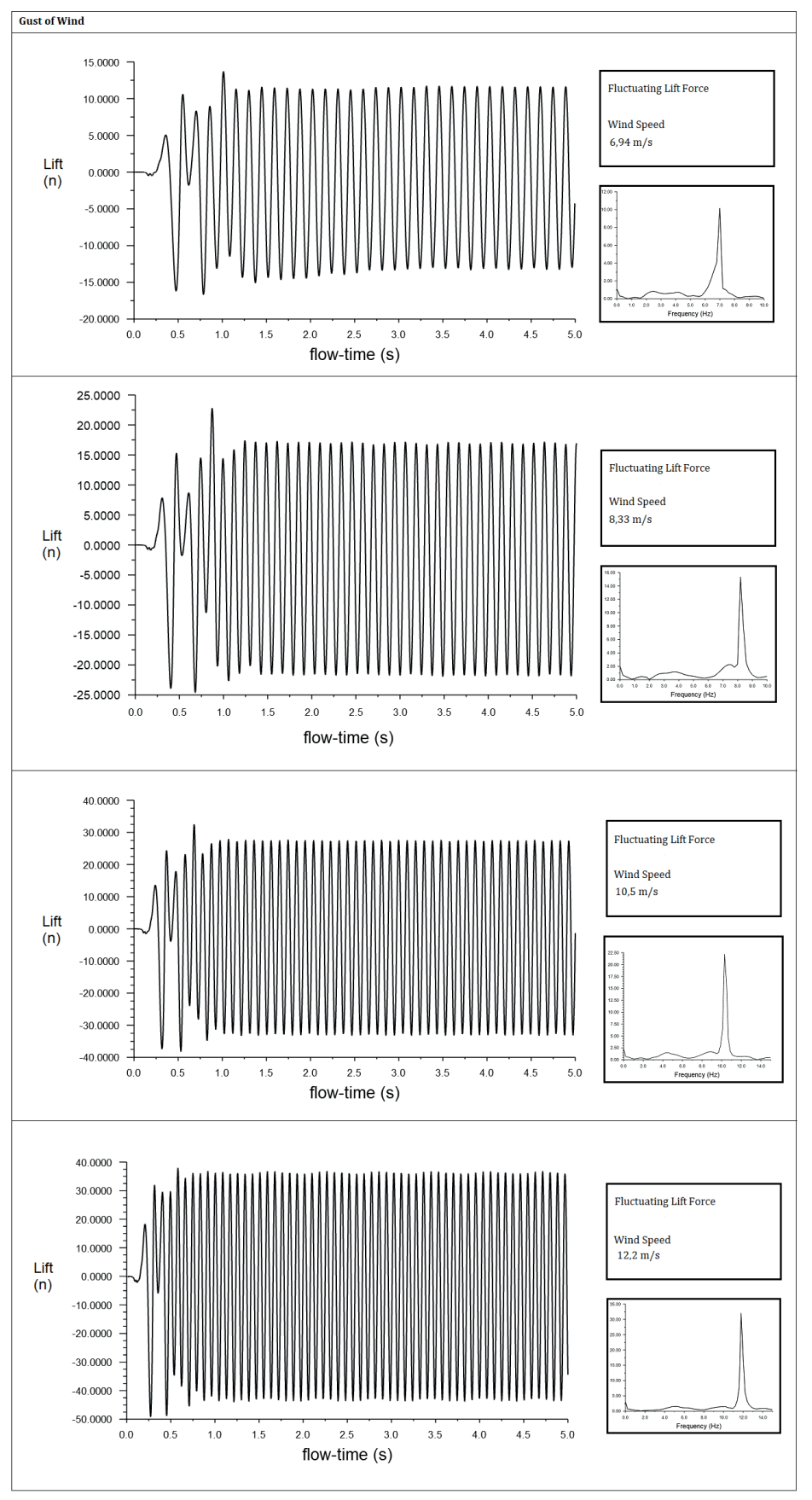


Para caracterizar la interacción fluido-estructura con los datos obtenidos, se ha establecido una fluctuación totalmente armónica de fuerza constante, con las magnitudes de oscilación en los transitorios, a lo largo de todo el mástil para así estudiar la respuesta armónica de la estructura con los diferentes modos de vibración natural. Para lograr sintonizar la frecuencia del desprendimiento de vórtices con la frecuencia natural, se ha parametrizado un soporte elástico variable en las zonas de amortiguamiento (figura 10) para ajustar la rigidez de toda la estructura. Estos soportes simulan el sistema de imanes fijos que sintonizan o ajustan las frecuencias para lograr resonancia.

Figura 10. Soporte elástico para ajuste de amortiguación y rigidez

\author{
F: Modal \\ Elastic Support \\ Frequency: N/A \\ $8 / 9 / 202111: 59$ \\ Elastic Support: 4,e-004 N/mm ${ }^{3}$
}
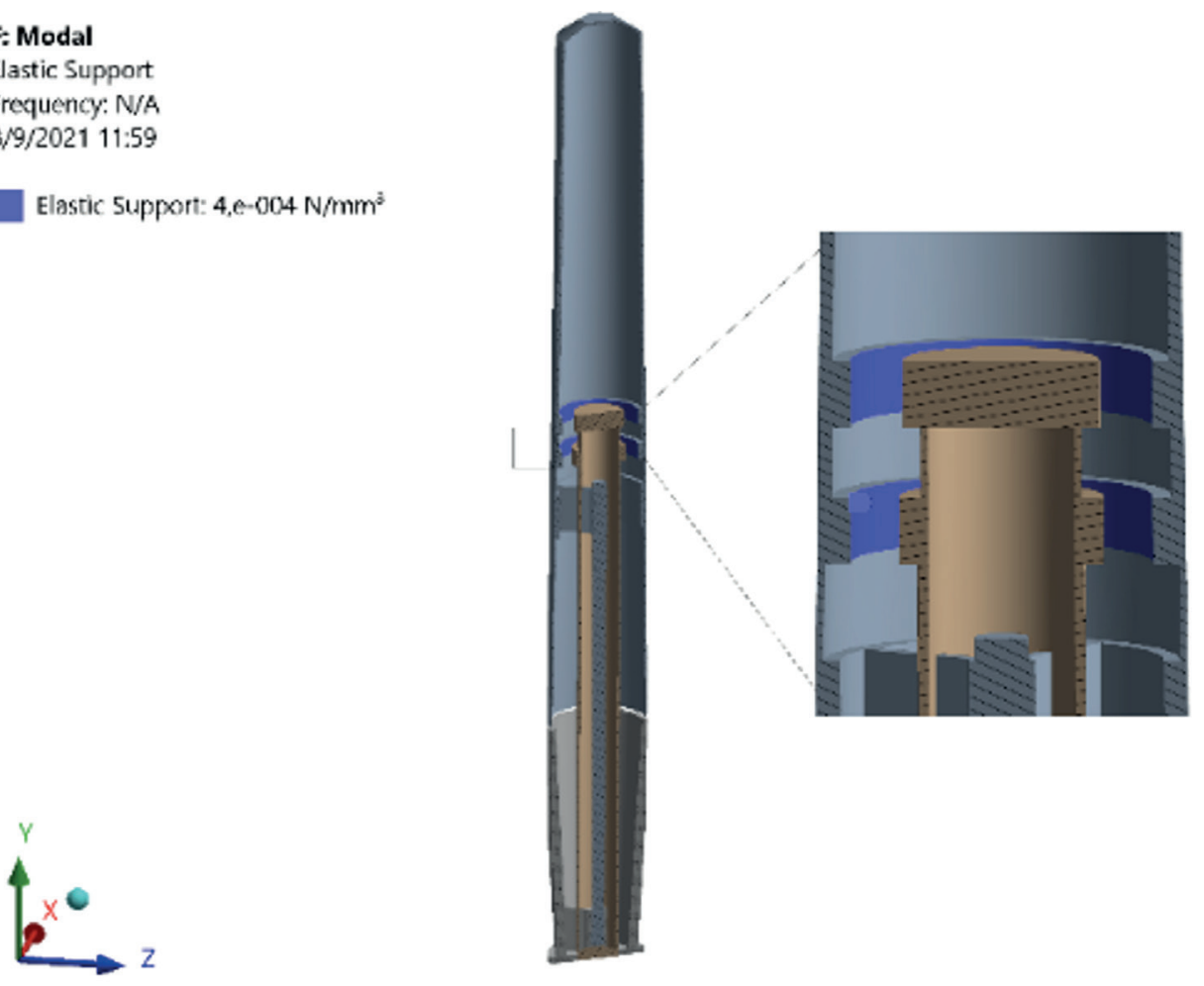

La rigidez necesaria para asemejar la frecuencia natural de la estructura a la de la excitación del viento varía según la intensidad en la que este fluye. En la tabla 2 constan las magnitudes en los soportes para sintonizar las frecuencias y así lograr resonancia. Analizando el desplazamiento vibratorio provocado por la oscilación, se puede ver que existe una disminución de la amplitud con la que vibra con el aumento de la rigidez hasta desaparecer a altas frecuencias. Esto es favorable para la dinámica del aerogenerador que trabaja en rangos bajos de velocidad del viento y se detiene con ráfagas muy altas evitando altas tensiones en la estructura y las fracturas. Utilizando el sistema de imanes fijos para amortiguar la estructura se puede automatizar el aumento de rigidez necesario para sintonizar la estructura con la excitación y alcanzar la resonancia. Esto se logra aprovechando las propiedades de respuesta entre dos imanes que se acercan con el mismo polo; es decir, la fuerza de repelencia es mayor, cuanto más se acercan el uno al otro (Domínguez Martinez et al., 2018). 
Tabla 2. Ajuste de la rigidez de la estructura para conseguir resonancia con la velocidad media del viento y las ráfagas de viento más frecuentes

\begin{tabular}{|c|c|c|c|c|}
\hline $\begin{array}{c}\text { Velocidad del } \\
\text { viento }\end{array}$ & $\begin{array}{c}\text { Frecuencia de } \\
\text { desprendimiento } \\
\text { de vórtice }\end{array}$ & $\begin{array}{c}\text { Magnitud del aumen- } \\
\text { to de la rigidez en los } \\
\text { soportes }\end{array}$ & $\begin{array}{c}\text { Frecuencia natu- } \\
\text { ral con soporte } \\
\text { de amortiguación }\end{array}$ & $\begin{array}{c}\text { Desplazamien- } \\
\text { to máximo en } \\
\text { resonancia }\end{array}$ \\
\hline \multicolumn{5}{|c|}{ Velocidad media } \\
\hline $2.77 \mathrm{~m} / \mathrm{s}$ & $2.62 \mathrm{~Hz}$ & $3.7 \mathrm{E}-4 \mathrm{~N} / \mathrm{mm}^{3}$ & $2.61 \mathrm{~Hz}$ & $130 \mathrm{~mm}$ \\
\hline \multicolumn{5}{|c|}{ Estalla } \\
\hline $6.94 \mathrm{~m} / \mathrm{s}$ & $7 \mathrm{~Hz}$ & $5 \mathrm{E}-2 \mathrm{~N} / \mathrm{mm}^{3}$ & $7.04 \mathrm{~Hz}$ & $118 \mathrm{~mm}$ \\
\hline $8.33 \mathrm{~m} / \mathrm{s}$ & $8.2 \mathrm{~Hz}$ & $0.12 \mathrm{~N} / \mathrm{mm}^{3}$ & $8.2 \mathrm{~Hz}$ & $102 \mathrm{~mm}$ \\
\hline $10.55 \mathrm{~m} / \mathrm{s}$ & $10.3 \mathrm{~Hz}$ & $0.29 \mathrm{~N} / \mathrm{mm}^{3}$ & $10.31 \mathrm{~Hz}$ & $70 \mathrm{~mm}$ \\
\hline $12.2 \mathrm{~m} / \mathrm{s}$ & $11.8 \mathrm{~Hz}$ & $0.43 \mathrm{~N} / \mathrm{mm}^{3}$ & $11.78 \mathrm{~Hz}$ & $62.8 \mathrm{~mm}$ \\
\hline
\end{tabular}

Como se esperaba y se puede ver en la tabla 2, es posible sintonizar la frecuencia excitante con la frecuencia natural variando la rigidez de la estructura. De esta forma se obtiene resonancia que se traduce en un movimiento vibratorio, el cual, a través de un alternador, puede transformarse en energía eléctrica para microconsumo. Con aerogeneradores de este tipo, pero más pequeños y de diferente mástil, se ha podido lograr entre 9 y $20 \mathrm{~W}$ para velocidades de 4 y $6 \mathrm{~m} / \mathrm{s}$ (Buela \& et al, 2021, junio 26). Vorttex Bladless presentó su aerogenerador Tacoma de $2.75 \mathrm{~m}$ con potencias estimadas de entre 0 y $100 \mathrm{~W}$ proporcionales a la potencia disponible del viento. La potencia disponible observada en el estudio está en un rango de $5.5 \mathrm{~W}-430 \mathrm{~W}$, que generan una oscilación con frecuencia media $7.21 \mathrm{~Hz}$ en la estructura y desplazamientos máximos de $13 \mathrm{~cm}$ con respecto al eje de inercia, con lo que se puede esperar una potencia similar a lo estimado de hasta $100 \mathrm{~W}$, por cada dispositivo puesto en marcha.

Figura 11. Esfuerzos equivalentes (Von Misses)

en la varilla de oscilación central causada por la vibración

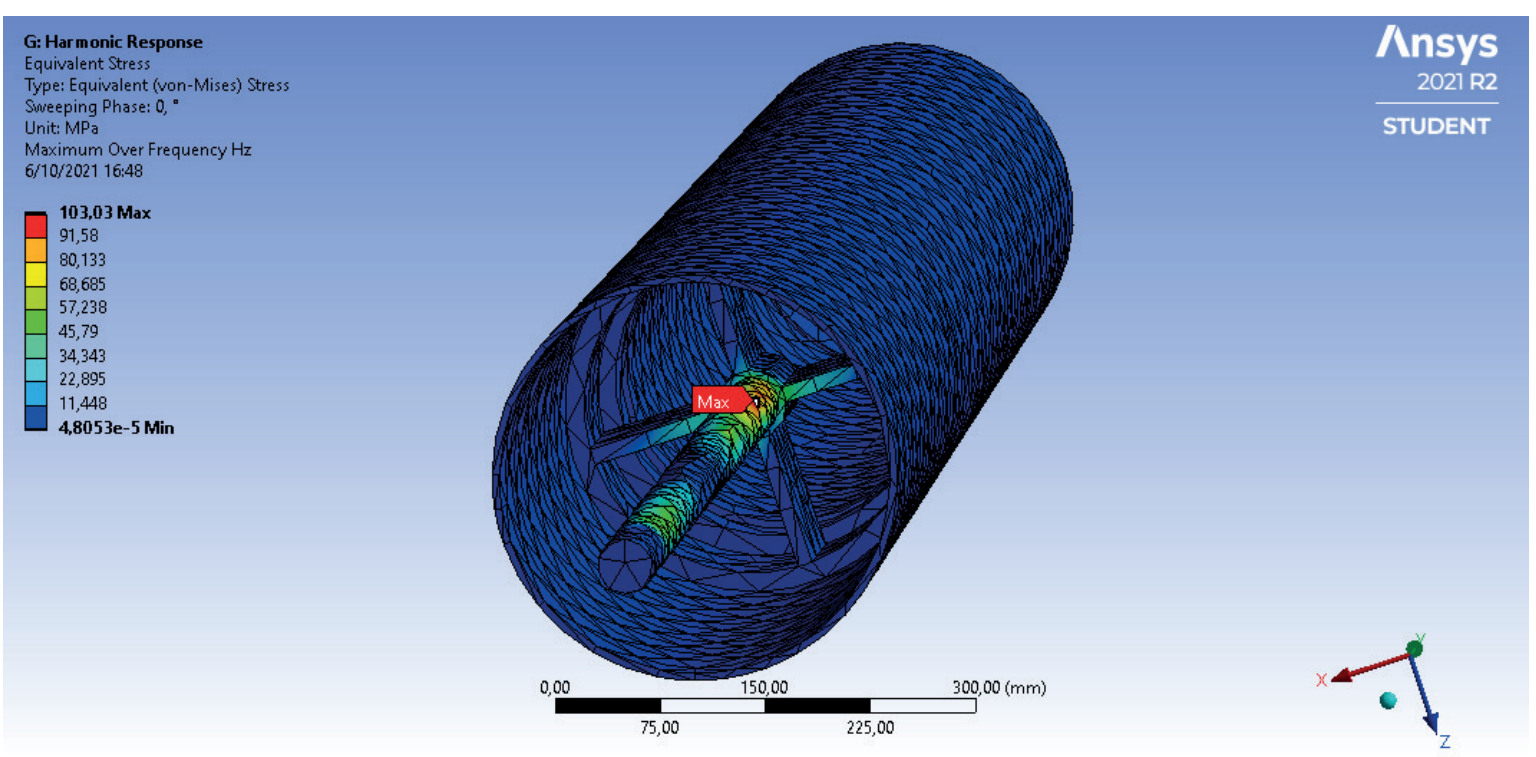


La respuesta de la estructura es parecida para los diferentes estímulos. Calibrando los soportes de amortiguación, puede definirse el rango de funcionamiento del aerogenerador para adaptarse a las condiciones ambientales donde operará. Mecánicamente, la varilla central de fibra de carbono (290 GPa) es la que recibe la carga y la única parte de la estructura que se deforma. Esta parte es la más propensa a fallar y fracturarse, sin embargo, esta varilla es fácil de reemplazar y no constituye un gasto mayor porque es una varilla delgada. En la figura 11 se ilustran las tensiones equivalentes de Von Mises que actúan sobre la varilla, las cuales mostrarían las áreas más afectadas por el movimiento y, por lo tanto, las posibles zonas de fractura.

\subsection{Influencia del entorno urbano en el desprendimiento de vórtices}

Utilizando la velocidad media del viento más frecuente, se ha analizado la influencia del entorno urbano, donde los edificios actúan como obstáculos al viento, lo cual afecta la interacción del fluido con la estructura. Con el modelo 2D transitorio se simula el flujo de viento sobre el aerogenerador instalado en un entorno con casas y edificios que intervienen en el mismo plano. Asimismo, se compara con el modelo no obstaculizado para determinar las variaciones más importantes que existen al instalar en un campo abierto y en el centro de una ciudadela. En la figura 12, en a) y b) se muestran los contornos de vorticidad en diferentes momentos para la simulación de campo abierto, donde se puede ver una dirección constante de desprendimiento de vórtices; mientras que en c) y d) constan el flujo con obstáculos a través del tiempo, y donde se ve cómo varía la dirección de la calle de vórtices.

Figura 12. Contornos de vorticidad para un flujo de viento de $2.77 \mathrm{~m} / \mathrm{s}$

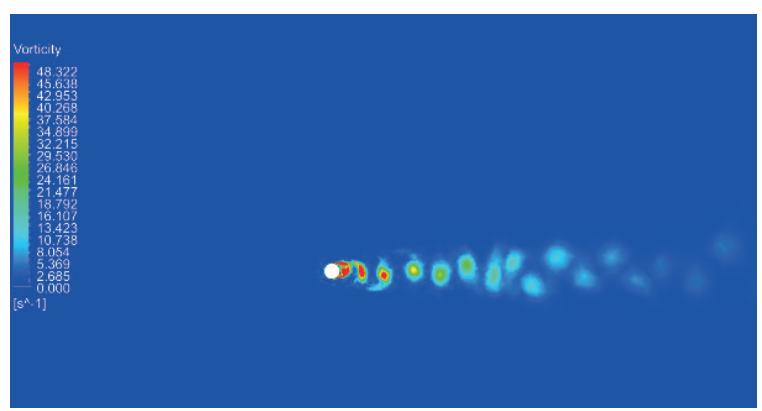

a) $t=5(s)$

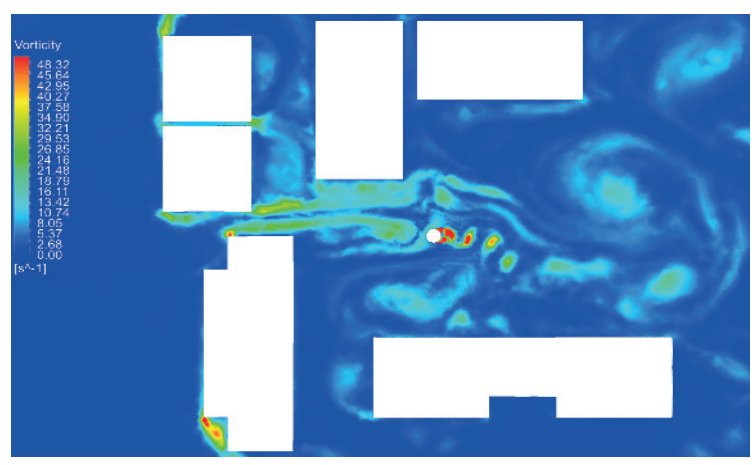

c) $t=5(s)$

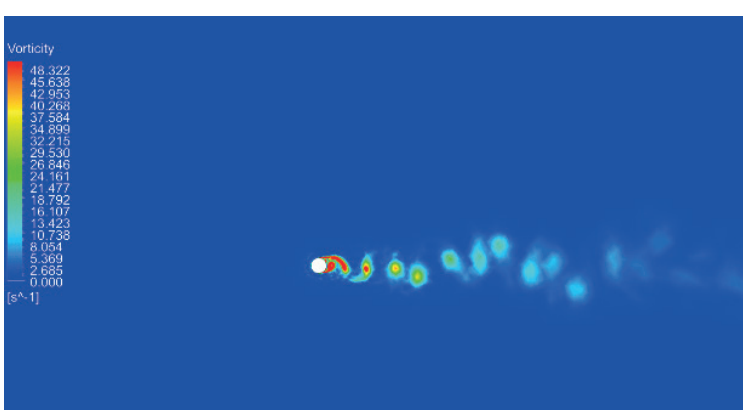

b) $t=10(s)$

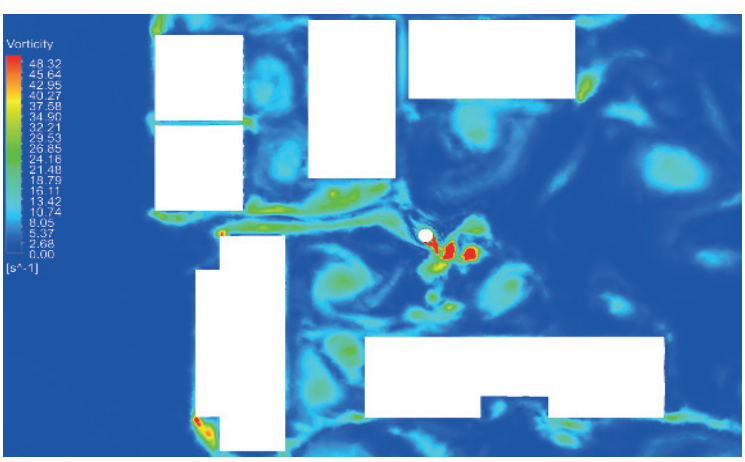

d) $t=10(\mathrm{~s})$

Nota: a) flujo libre a t= $5 \mathrm{~s}$, b) flujo libre a t= $10 \mathrm{~s}$, c) flujo con obstáculos a t= $5 \mathrm{~s}$ y d) flujo con obstáculos a t= $10 \mathrm{~s}$. 
La principal variación que existe entre los resultados de los dos modelos es la causada por obstáculos en la dirección del flujo del viento que altera directamente la dirección de la calle de vórtices en la estela dejada por el viento al cruzar la estructura del aerogenerador, a diferencia de un flujo sin obstáculos donde la dirección es constante si la dirección del viento entrante no cambia. También se puede ver que estos efectos alteran la frecuencia de desprendimiento de vórtice, con lo que en el estudio CFD no se ha podido determinar una fluctuación constante 0 armónica de la excitación causada por el viento principalmente porque la calle de vórtices no actúa sobre un solo eje fijo; sin embargo, se ha observado si estos efectos han sido favorables para la magnitud de la fuerza de elevación. Al ser una estructura semicilíndrica, las alteraciones en la dirección del viento no modifican su funcionamiento, porque está diseñada para trabajar de la misma manera, sea cual sea la dirección de este. De este modo, el dispositivo puede aprovechar los flujos turbulentos y las ráfagas de viento que se generan en un entorno urbano, así como trabajar en conjunto varios de estos dispositivos, porque su influencia aguas abajo es despreciable como se vio en las simulaciones dinámicas, aspecto que también es favorable para la fauna urbana.

\section{Conclusiones}

El aerogenerador sin palas aprovecha un efecto transitorio de dinámica de fluidos llamado von Karman vortex street, el cual genera una señal fluctuante en la dirección transversal a la del flujo de viento. Esta señal tiene una frecuencia característica que al acercarse a la frecuencia natural de la estructura hará que vibre y genere energía mecánica. El aerogenerador sin palas convierte esta energía mecánica en pequeñas cantidades de energía eléctrica que pueden ser utilizadas en hogares, ya sea en entornos rurales o urbanos, ajustando las condiciones de funcionamiento para aprovechar los fenómenos estocásticos que se producen en función del lugar de instalación. Junto con otros sistemas de energía renovable, es posible hacer que las ciudades y pueblos sean autosostenibles, porque consumen la misma energía que producen en el lugar donde se encuentran.

Con el fin de alcanzar el desarrollo sostenible, se busca generadores de energía eléctrica que no contaminen y que sean más eficientes a los convencionales. El aerogenerador por vibraciones inducidas es una alternativa que utiliza energía renovable del viento y no perturba significativamente el entorno donde está instalado. Funciona en condiciones de viento muy bajo, como se pudo ver en el estudio fluido-estructura, y también funcionaría correctamente en condiciones de viento cotidianas, y no es necesario que haya velocidades de viento muy altas. El aerogenerador trabaja en magnitudes de entre $2.77 \mathrm{~m} / \mathrm{s}$ a aproximadamente $12 \mathrm{~m} / \mathrm{s}$ aprovechando la velocidad media del viento y las rachas o ráfagas de viento que se produzca durante el día. Como se pudo constatar, la frecuencia de oscilación crece proporcionalmente al aumento de la velocidad, así como a la fuerza de elevación.

Mecánicamente, la estructura estudiada con base en el modelo de 2.75 m y diámetro nominal entre 0.3 y 0.25 m mostró condiciones adecuadas para generar energía con las condiciones ambientales en los 7 días que se recogieron los datos. La respuesta a los armónicos provocada por el desprendimiento de vórtices de viento midió una distancia de oscilación de hasta $13 \mathrm{~cm}$ con respecto al eje central fijo con frecuencias entre 2.62 y $11.8 \mathrm{~Hz}$, oscilaciones que, teóricamente, podrían generar hasta $100 \mathrm{~W}$ en ráfagas de viento. Todo el esfuerzo mecánico es absorbido por la varilla central conectada al mástil, la cual alcanza tensiones equivalentes 
de entre 40 y 70 MPa. Esta es la única parte que sufre deformaciones, por lo tanto, es la más propensa a fracturas y fallos.

Analizando la influencia de un área urbana con edificios pequeños en la aerodinámica del dispositivo, se pudo ver, en comparación con un área sin obstrucciones, que la dirección del viento se altera por periodos. Esto hace que la dirección de oscilación de la turbina eólica también cambie; aumenta la turbulencia en el flujo del viento, lo cual altera la amplitud de la señal de excitación y la fuerza de elevación que actúa. Sin embargo, estos cambios no alteran significativamente el funcionamiento del aerogenerador, porque se trata de una estructura semicilíndrica omnidireccional que funciona correctamente en todas las direcciones del viento, por lo tanto, constituye una gran ventaja para un dispositivo que debe instalarse en ambientes urbanos con muchas obstrucciones al fluir del viento.

\section{Referencias}

Andrade, A. (2021, julio 27). Objetivos de Desarrollo Sostenible en Ecuador. Investoria Fundation. https:// bit.ly/300YGi4

Ansys, I. C. (2020). Formulation of harmonic analysis: Introduction, complex variables and notation, displacement-velocity-acceleration, formulation and derivation, discussion. ANSYS. https://bit.ly/3KQJJcf

Buela, A., Rey, R., Unisa, F., Meris, P., Manuel, M., De la Cruz, J., \& Tud, R. (2021, junio 26). Design and nonlinear static simulation of a small-scale vortex bladeless wind power generator. 2021 IEEE International Conference on Automatic Control \& Intelligent Systems, Shah Alam, Malasia 185-190. https://doi.org/10.1109/I2CACIS52118.2021.9495882

Bustamante Campoverde, A. S. (2021). Caracterización del viento y temperatura aparente en los cañones urbanos del centro histórico de Cuenca, Ecuador. Conservar Património, 36, 90-105. https:// doi.org/10.14568/cp2019034

Comisión de Sustentabilidad Capbauno. (2020). Ciudades sostenibles. Capbauno Obtenido de http:// www.capbauno.org.ar/

Domínguez Martinez, M., Toral, F., Ghasem, H., Papadopoulou, P. S., \& Papaphilippou, Y. (2018). Longitudinally variable field dipole design using permanent magnets for clic damping rings. IEEE Transactions on Applied Superconductivity, 28(3), 1-4. https://doi.org/10.1109/TASC.2018.2795551

Guevara Díaz, J. (2013). Cuantificación del perfil del viento hasta $100 \mathrm{~m}$ de altura desde la superficie y su incidencia en la climatología eólica. Terra, 29(46), 81-101. http://ve.scielo.org/scielo. php?script=sci_arttext\&pid=\$1012-70892013000200006

Hu, J., Wang, Z., Zhao, W., Sun, S., Sun, C., \& Guo, C. (2020). Numerical simulation on vortex shedding from a hydrofoil in steady flow. Journal of Marine Science and Engineering, 8(3), 195. https:// doi.org/10.3390/jmse8030195

Hu, Y., Yang, B., Chen, X., Wang, X., \& Liu, J. (2018). Modeling and experimental study of a piezoelectric energy harvester from vortex shedding-induced vibration. Energy Conversion and Management, 162, 145-158. https://doi.org/10.1016/j.enconman.2018.02.026

Karaağaçlı, T., \& Özgüven, N. (2021). Experimental modal analysis of nonlinear systems by using responsecontrolled stepped-sine testing. Mechanical Systems and Signal Processing, 146. https://doi. org/10.1016/j.ymssp.2020.107023

López, O. D. (2002). Modelamiento computacional de la calle de vértices de karma por dinámica de verticidad. Mecánica Computacional, XXI, 274-292.

Meteored. (2021). Histórico del tiempo en Quito. Quito - Aeropuerto Mariscal Sucre Intl (SEQU): Meteored.

Peter, S., \& Leine, R. (2017). Excitation power quantities in phase resonance testing of nonlinear systems with phase-locked-loop excitation. Mechanical Systems Signal Processign, 96, 139-158. https://doi.org/10.1016/j.ymssp.2017.04.011 
Saengsaen, S., Chantharasenawong, C., \& Wu, T.-L. (2019). A 2-d mathematical model of vortex induced vibration driven bladeless wind turbine. MATEC Web of Conferences, 291, artículo 02007. https://doi.org/10.1051/matecconf/201929102007

Spalart, P. R., \& Allmaras, S. R. (1992). A one-equation turbulence model for aerodynamic flows. La Recherche Aérospatiale, 1, 5-21. https://doi.org/10.2514/6.1992-439

Yáñez, D. J. (2018, enero 23). España Patente n BR112013002403A.

Yáñez, D. J. (2018, junio 7). Aerogeneradores resonantes por VIV. Vortex Bladeless S. L. https://vortexbladeless.com/?smd_process_download=1\&download_id $=4390$ 